\title{
Reforma de Salud en Chile: Evaluación Garantía Explícita en Salud Oral en Población de 60 Años
}

\author{
Evaluation of Explicit Guarantees in Oral Health in 60-year-old Population
}

Cristóbal Quezada Peña*; Daniela Muñoz Ávila*; Alfredo Cueto Urbina"** \& Alan Barraza Sáez

QUEZADA, P. C.; MUÑOZ, A. D.; CUETO, U. A. \& BARRAZA, S. A. Reforma de salud en chile: Evaluación Garantía Explícita en Salud Oral en Población de 60 años. Int. J. Odontostomat., 7(2):319-326, 2013.

RESUMEN: La reforma chilena creó el Programa de Garantías Explícitas en Salud (GES) para salvaguardar la atención bajo condiciones garantizadas de acceso, oportunidad, protección financiera y calidad para situaciones prioritarias como la salud oral del adulto de 60 años. Objetivo: Comparar la salud bucodental y satisfacción entre pacientes beneficiarios del GES salud oral integral 60 años y no beneficiarios. Estudio caso-control. Los participantes se dividieron en casos $(n=48)$ y grupo control $(n=28)$. Examen clínico recogiendo las variables: sexo, edad, enfermedades sistémicas declaradas, nivel educacional, uso de prótesis, índices COPD, CPITN y O’Leary. Para medir satisfacción se utilizó la escala multidimensional SERVPERF no ponderado. Análisis inferencial mediante test de significancia (pruebas de Pearson, Exacto de Fisher, Mann-Whitney, Medianas de Mood y Kolmogorov-Smirnov con un nivel de confianza del 95\%) en los software SPSS19, Stata-SE 10.1 y Minitab 15. En las mediciones bucodentales se encontraron diferencias significativas sólo en cariados y COPD. No hubo diferencias en las necesidades de tratamiento periodontal entre los grupos ( $p$-valor=0.615). La presencia de prótesis removible es estadísticamente mayor en el grupo de casos $(O R=4,4$ [1,6-12,5]); $p$-valor=0,006). La reforma en salud a través del GES Salud Oral Integral de 60 años, demuestra variar los parámetros clínicos de los pacientes beneficiarios, enfocado a la restauración con un aumento significativo de prótesis y una disminución de las caries, pero no logra mantener la salud periodontal ni el manejo de los factores de riesgo. La satisfacción del usuario es buena en los beneficiarios del GES.

PALABRAS CLAVE: programas salud bucal, ancianos, bucal, diagnóstico, servicio dental para ancianos, satisfacción del paciente.

\section{INTRODUCCIÓN}

Según la CEPAL, en los próximos años la tasa de crecimiento de la población mayor de 60 años será de un $4 \%$ anual para toda Latinoamérica (CELADE, 2010). En Chile al año 2010, la población mayor de 60 años alcanzaba el 13\% (INE, 2010), lo que se clasifica como población envejecida, para enfrentar este desafío se implementó a partir del 2007 la Reforma en Salud.

La reforma creó el Programa de Garantías Explícitas en Salud (GES), el cual salvaguarda la atención bajo condiciones garantizadas de acceso, oportunidad, protección financiera y calidad de atención en salud, para un conjunto de situaciones de salud prioritarias, entre estas la salud oral del adulto de 60 años (MINSAL, 2011). Desde su implementación en el año 2005, un total de 11.864.093 personas han utilizado la reforma en las 69 prestaciones que cubre el GES; de éstas, un total acumulado desde Julio del 2007 a Marzo del 2011 de 101.641 adultos mayores de 60 años fueron beneficiados con el GES salud oral integral (Superintendencia de Salud, 2011a).

Siendo Chile un país con grandes desigualdades sociales, resulta relevante evaluar las políticas de salud implementadas en especial estas garantías que han significado grandes esfuerzos económicos y humanos en su puesta en marcha. Y por otro lado, es

\footnotetext{
Cirujano dentista. Universidad de Valparaíso, Valparaíso, Chile.

* Cirujano dentista. Profesora ayudante de la Facultad de Odontología, Universidad de Valparaíso, Valparaíso, Chile.

** Cirujano dentista. Profesor Titular de la Facultad de Odontología, Universidad de Valparaíso, Valparaíso, Chile.

Estat Estástico. Profesor ayudante de la Facultad de Odontología, Universidad de Valparaíso, Valparaíso, Chile.
} 
importante conocer la percepción de los beneficiarios, pues uno de los objetivos sanitarios para la década 2000-2010 consistió en "Prestar servicios sanitarios acordes a las expectativas de la población" (MINSAL, 2002). La evaluación de este programa podría contribuir a que las autoridades en salud de otros países de Latinoamérica, ante una eventual implementación de programa similar puedan aprovechar esta experiencia.

El propósito de esta investigación es conocer el efecto de las GES en la salud oral del paciente de 60 años determinando su nivel de salud y su grado de satisfacción.

El objetivo fue comparar la salud bucodental y grado de satisfacción entre pacientes beneficiarios del GES salud oral integral 60 años y no beneficiarios entre 61 y 69 años.

\section{MATERIAL Y MÉTODO}

Estudio de caso-control (Hernández Sampieri, 2006). El universo de este estudio es la población beneficiaria de la salud pública de los centros de salud de Valparaíso (la tercera comuna más habitada de Chile). Se trabajó con una muestra mínima para detectar diferencias entre grupos utilizando los datos del total de inscritos mayores de 60 años (13.033) obteniéndose un grupo beneficiado (casos) de 48 personas y otro grupo (controles) de pacientes atendidos durante el 2010 (no necesariamente de alta) de 28 personas entre 61 y 69 años. Los individuos se seleccionaron de manera aleatoria de acuerdo a la proporción de cada centro de salud en la población total (CORMUVAL, 2009). El protocolo de la investigación fue aprobado por el Comité de Ética institucional (Resolución 201010N04) y se obtuvo el consentimiento informado de todos los participantes. Se les examinó clínicamente para recoger las siguientes variables: sexo, edad, enfermedades sistémicas declaradas, nivel educacional, índice COPD (cariados, obturados y perdidos por diente), índice CPITN (Community Periodontal Index of Treatment Needs), Índice O'Leary y uso de prótesis. El examen utilizó bandeja de examen, sonda de caries, pinza, espejo, sonda OMS; revelador de placa, iluminación natural,vaciando los datos en una ficha de recolección construida según recomendación de la OMS (1997). Para medir la satisfacción se utilizó la escala multidimensional SERVPERF no ponderado. Este instrumento (encuesta) se basa en la calidad de servicio percibido y para ello utiliza 4 dimensiones para medir esta percepción, las primeras 3 se refieren a características generales de cualquier servicio y son las siguientes: confiabilidad (habilidad para ejecutar el servicio prometido de forma fiable y cuidadosa), capacidad de respuesta (voluntad, disposición a ayudar y proveer un servicio rápido) y elementos tangibles (Infraestructura). La cuarta dimensión consideró evaluar la percepción acerca de las condiciones garantizadas en el acceso, oportunidad, financiamiento y calidad del servicio odontológico. Cada dimensión consta de 4 afirmaciones que se calificaron mediante escala Likert (1=malo, $2=$ regular y $3=$ bueno).

La recolección de datos se realizó con un encuestador/examinador debidamente calibrado (Kappa=0,8).

Se hizo un análisis inferencial a través de test de significancia utilizando las pruebas de Pearson, Exacto de Fisher, Mann-Whitney, Medianas de Mood y Kolmogorov-Smirnov para dos muestras independientes con un nivel de confianza del $95 \%$, para evaluar y comparar los grupos de estudio, se utilizaron los software SPSS Versión 19, Stata-SE 10.1 y Minitab 15.

\section{RESULTADOS}

La comparación entre casos y controles en múltiples variables se observa en la Tabla I, resultando similares comportamientos entre ambos grupos, excepto para estado civil y diabetes.

Al comparar los casos y los controles en variables odontológicas que se observan en la Tabla II, se constatan diferencias significativas sólo en cariados y COPD.

Al comparar el índice periodontal CPITN los códigos resultantes de su medición se agrupan en dos categorías de acuerdo a la severidad de los signos de enfermedad periodontal (ver Tabla III), en ésta no se encontraron diferencias en las necesidades de tratamiento periodontal entre los grupos (Test exacto de Fisher: $\mathrm{p}$-valor $=0,615)$. Los pacientes sin signos de enfermedad periodontal fueron $3(6,8 \%)$ en los casos y 1 $(3,7 \%)$ en los controles.

Al evaluar el edentulismo en el maxilar inferior, en los casos alcanzó el $97,9 \%$ y en los controles fue de $96,4 \%(p$-valor $=0,99)$ y para el maxilar superior, 
QUEZADA, P. C.; MUÑOZ, A. D.; CUETO, U. A. \& BARRAZA, S. A. Reforma de salud en chile: Evaluación Garantía Explícita en Salud Oral en Población de 60 años. Int. J. Odontostomat., 7(2):319-326, 2013.

Tabla I. Características de los grupos.

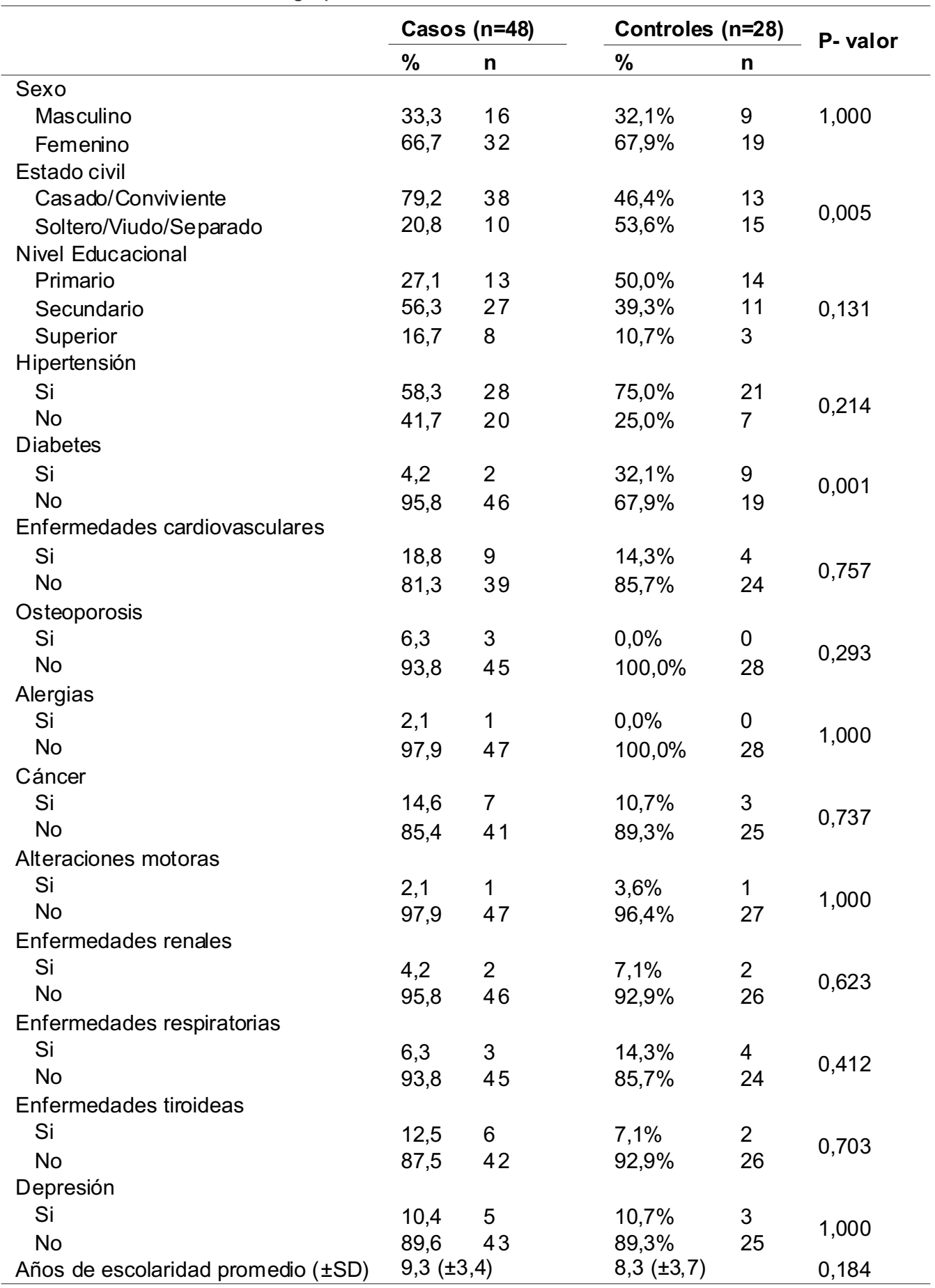

en los casos $100 \%$ y en los controles fue de $96,4 \%$ (pvalor $=0,74$ ). Para ambas comparaciones no se apreciaron diferencias significativas entre los grupos de estudio. Sin embargo, al evaluar la proporción de personas que poseen prótesis removible (ver Tabla IV) es estadísticamente mayor en el grupo de casos (Test exacto de Fisher: P-valor=0,006), se estima que la presencia de prótesis removible es 4,4 (IC 95\% OR = $[1,6-12,5])$ veces más probable en los casos que en controles. 


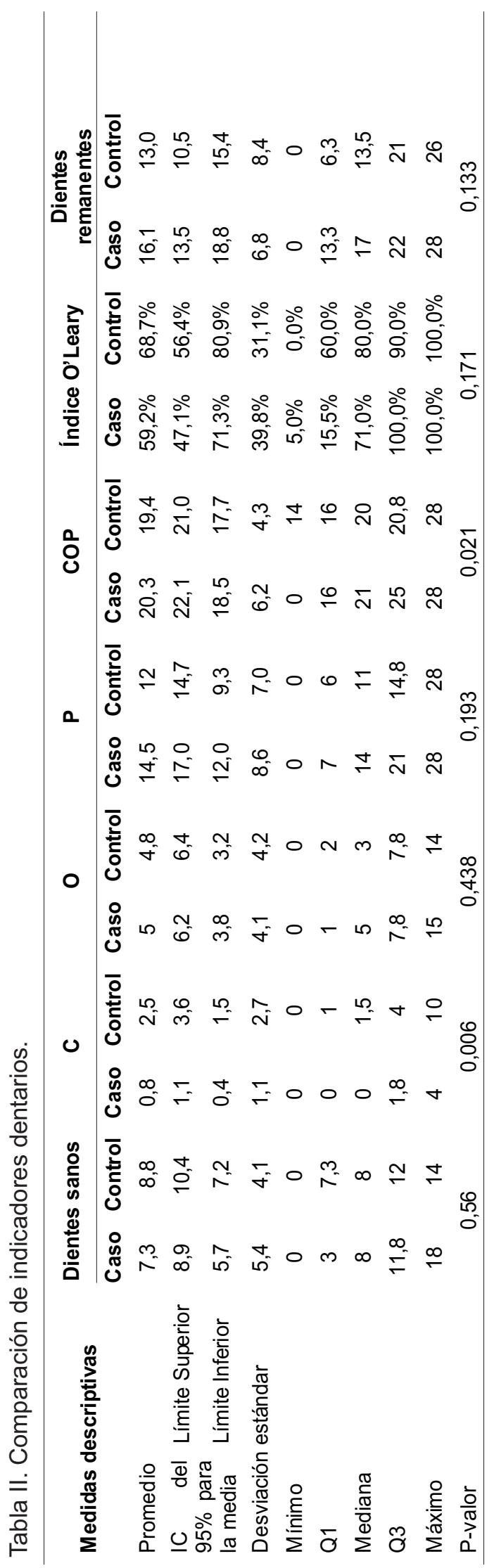

Tabla III. Índice de necesidad de tratamiento periodontal de la comunidad (CPITN) por grupo.

\begin{tabular}{llllll}
\hline & \multicolumn{2}{c}{ Casos $(\mathrm{n}=48)$} & & \multicolumn{2}{l}{$\begin{array}{l}\text { Controles } \\
(\mathrm{n}=28)\end{array}$} \\
\cline { 2 - 3 } \cline { 6 - 7 } Código CPITN & $\mathrm{n}$ & $\%$ & & & $\%$ \\
\hline Códigos 0, 1 y 2 & 14 & 31,8 & & 7 & 25,9 \\
Códigos 3 y 4 & 30 & 68,1 & & 20 & 74,0 \\
\hline
\end{tabular}

Tabla IV. Presencia de prótesis removible.

\begin{tabular}{|c|c|c|c|c|c|c|}
\hline \multirow{2}{*}{$\begin{array}{l}\text { Presencia } \\
\text { removible }\end{array}$} & \multirow{2}{*}{ de } & \multirow{2}{*}{ prótesis } & \multicolumn{2}{|c|}{$\begin{array}{l}\text { Casos } \\
(n=48)\end{array}$} & \multicolumn{2}{|c|}{$\begin{array}{l}\text { Controles } \\
(n=28)\end{array}$} \\
\hline & & & $n$ & $\%$ & $\mathrm{n}$ & $\%$ \\
\hline $\mathrm{Si}$ & & & 37 & 77,1 & 12 & 42,9 \\
\hline No & & & 11 & 22,9 & 16 & 57,1 \\
\hline
\end{tabular}

La variable satisfacción usuaria según las diferentes dimensiones se observa en la tabla V.

Como se observa, a través de todas las dimensiones de la encuesta de satisfacción, los pacientes en ambos grupos perciben una calidad del beneficio en salud como mayoritariamente de buena forma, con mayor tendencia hacia bueno en los pacientes GES. En las dimensiones de confiabilidad, tangibilidad y valoración GES si se observan diferencias significativas en la distribución de respuestas entre los grupos, siendo la proporción de repuestas positivas (Bueno) estadísticamente mayor en el grupo Beneficiados GES.

Al analizar el nivel de satisfacción de los usuarios GES y no GES, en la Figura 1 de análisis de correspondencia, la categoría Bueno es la más cercana a las cuatro dimensiones de la calidad en beneficiarios GES.

\section{DISCUSIÓN}

Los principales hallazgos de este estudio fueron que el programa GES en la práctica se traduce en actividades de rehabilitación disminuyendo el promedio de cariados del índice COPD y, por otro lado, aumenta sustantivamente los tratamientos protésicos de tipo removible. Sinembargo, no demuestra capacidad para mejorar el daño periodontal ni los niveles de higiene.

Desde el punto de vista de la satisfacción del usuario, se eleva a un nivel bueno, en especial en la dimensión de valoración del GES que reconoce acceso, oportunidad, financiamiento y calidad en la atención. 
Tabla V. Distribución de respuestas por categoría, dimensión y grupos.

\begin{tabular}{|c|c|c|c|c|c|c|c|c|c|}
\hline \multirow{2}{*}{ Dimensión } & & \multirow{2}{*}{$\begin{array}{l}\text { Conteo de } \\
\text { respuestas }\end{array}$} & \multicolumn{3}{|c|}{ Casos $(n=48)$} & \multicolumn{3}{|c|}{ Controles $(n=28)$} & \multirow{2}{*}{ P-valor } \\
\hline & & & Malo & Regular & Bueno & Malo & Regular & Bueno & \\
\hline \multirow{2}{*}{ Confiabilidad } & & $\mathrm{n}$ & 5 & 17 & 170 & 11 & 16 & 85 & \multirow{2}{*}{0,005} \\
\hline & & $\%$ & $2,6 \%$ & $8,9 \%$ & $88,5 \%$ & $9,8 \%$ & $14,3 \%$ & $75,9 \%$ & \\
\hline \multirow{2}{*}{$\begin{array}{l}\text { Capacidad } \\
\text { respuesta }\end{array}$} & de & $\mathrm{n}$ & 13 & 12 & 167 & 10 & 12 & 90 & \multirow{2}{*}{0,274} \\
\hline & & $\%$ & $6,8 \%$ & $6,3 \%$ & $87,0 \%$ & $8,9 \%$ & $10,7 \%$ & $80,3 \%$ & \\
\hline \multirow{2}{*}{ Tangibilidad } & & $\mathrm{n}$ & 3 & 12 & 177 & 5 & 14 & 93 & \multirow{2}{*}{0,045} \\
\hline & & $\%$ & $1,6 \%$ & $6,3 \%$ & $92,1 \%$ & $4,5 \%$ & $12,5 \%$ & $83,0 \%$ & \\
\hline \multirow{2}{*}{ Valoración GES } & & $\mathrm{n}$ & 6 & 6 & 180 & 4 & 13 & 95 & \multirow{2}{*}{0,012} \\
\hline & & $\%$ & $3,1 \%$ & $3,1 \%$ & $93,7 \%$ & $3,6 \%$ & $11,6 \%$ & $84,8 \%$ & \\
\hline
\end{tabular}
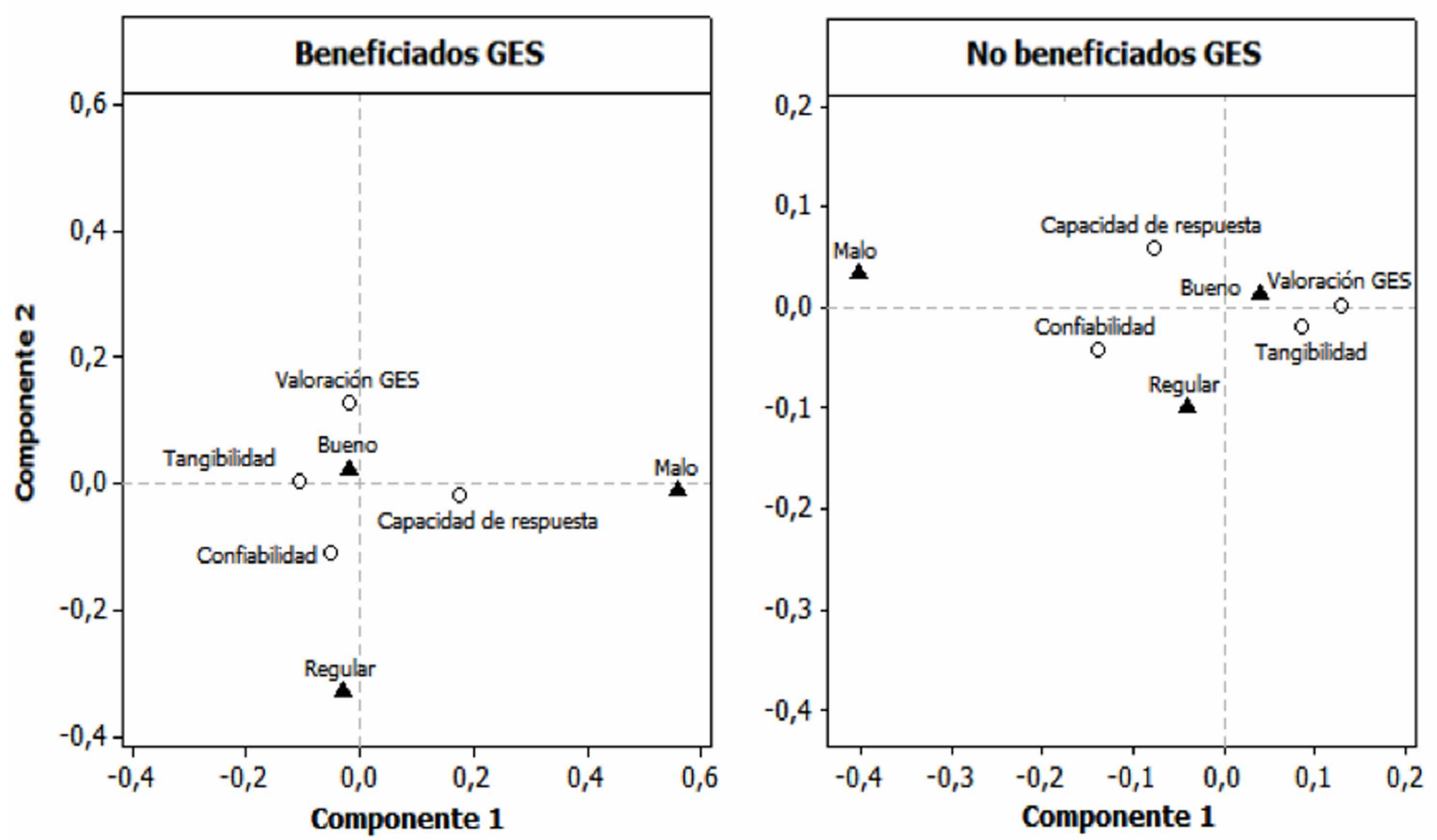

Fig. 1. Proyección de frecuencias de respuesta en ambos grupos estudio.

Este estudio se realizó en Valparaíso, una de las diez comunas más pobladas de Chile, con una considerable población de adultos mayores (13\%) (Moreno \& Miles, 2002), lo que permitirá hacer inferencia para el resto del país.

Las encuestas de salud bucodental en sus métodos básicos, han sido definidos por la OMS. Este estudio se ajustó a dichas recomendaciones en cuanto a las variables bucodentarias recogidas (entre éstas los índices COPD y CPITN). La escala SERVPERF es de reconocido uso internacional, investigaciones previas (Cronin \& Taylor, 1994) han concluido que medir la calidad del servicio basada en percepciones SERVPERF no ponderado es más apropiada que otros instrumentos. 
Los grupos estudiados se comportaron en forma similar en las variables de carácter general como: años de escolaridad, nivel educacional, género con predominio femenino y antecedentes mórbidos, haciendo pertinente la comparación desde el punto de vista de la salud oral.

El índice COPD promedio de los casos fue 20,2 y 19,3 para los controles, ambos resultaron menores al reportado en un estudio de prevalencia efectuado en población mayor en Valparaíso (Mariño et al., 2011), el cual informó un COPD promedio de 21,6 en 354 adultos mayores de 65 a 74 años. La diferencia se podría atribuir a la edad promedio pues en nuestro estudio fue menor al citado (Mariño et al.), además existen diferencias del nivel de ruralidad de las poblaciones examinadas.

El índice COPD en los casos y los controles es muy alto, lo que refleja un gran deterioro de la salud bucodental, esto se explica en gran parte porque reflejan un daño acumulado para esa edad, pues los adultos mayores no tuvieron, durante su juventud, medidas públicas de promoción y prevención, ni menos programas que cubrieran tratamientos odontológicos adecuados por falta de recursos.

Se observó diferencia estadísticamente significativa en la cantidad de dientes cariados, esto se explica ya que el grupo casos tenía acceso privilegiado a la atención dental teniendo tratamiento garantizado en cuanto a acceso, oportunidad, financiamiento y calidad. Llama la atención no encontrar diferencias significativas en el porcentaje de obturados, atribuible a una acción odontológica más mutiladora que restauradora. De hecho el promedio de dientes perdidos es mayor en el grupo de los casos que en el grupo control.

Los pacientes controles podrían haber tenido acceso a otros programas de atención odontológica como el Programa de Reparación y Atención Integral de Salud a las víctimas de represión política por parte del Estado, de hecho un porcentaje no menor $(50,0 \%)$ declaró utilizar el sistema público y privado. Todas estas posibilidades de acceso a tratamiento pueden están influyendo en disminuir las diferencias en la situación de salud oral de estos grupos.

La necesidad de tratamiento periodontal resultó ser la más prevalente en el adulto mayor, encontrándose sólo un $6,8 \%$ en el grupo de casos, y aún menor en los controles, sin signos de enfermedad, en poblaciones comparables el 2006, para la región de Valparaíso se reportó un 3,3\% de ausencia de signos (Badenier et al., 2007) y en países con mayor desarrollo reportan 8,7\% (Petersen et al., 2007). Estos resultados pueden explicarse en parte debido a los altos índices de placa (O'Leary) hallados en el presente estudio pues para que un tratamiento periodontal sea efectivo debe ir acompañado del control del biofilm por parte del paciente (Mariño, 1994), además, las enfermedades periodontales pueden ser refractarias al tratamiento en pacientes con antecedentes sistémicos de importante prevalencia en la población adulta mayor del estudio, para asegurar el éxito del tratamiento periodontal debe existir seguimiento y control, que este programa no proporciona (Rojas et al., 2010).

Como probable explicación al deterioro de la salud oral de esta población algunos autores han señalado que "es posible que el adulto mayor considere natural perder los dientes progresivamente hasta llegar a la pérdida total" (Helft et al., 1986). Esto influiría en que los pacientes de edad avanzada descuiden las medidas preventivas necesarias para mantener la salud de las encías14. Sin embargo, "se sabe que es posible mantener la buena salud orodental hasta edad avanzada y que la dentadura no se pierde a causa del envejecimiento, sino debido a enfermedades orodentales asociadas con diversos factores de riesgo" (Mariño). Este programa de salud oral integral 60 años considera actividades de prevención y hasta de rehabilitación, pero debe profundizar en estrategias efectivas para lograr cambios de conductas pues lo que se hace actualmente resulta insuficiente.

Al examinar los pacientes, en especial su estado periodontal, con el índice CPITN, no se encontraron diferencias significativas entre los grupos; es probable que esta falta de diferencia pueda atribuirse a que el grupo casos ha tenido recidiva de la enfermedad periodontal, por tanto el programa GES no es efectivo para controlar los factores de riesgo y por tanto se hace necesario mejorar la implementación del GES en este punto, se podría reforzar periódicamente las indicaciones de cuidados de la salud oral, en especial técnicas de higiene, lograr experiencias educativas significativas, e incorporar controles periódicos eventualmente asociados a un estímulo económico como han funcionado otros programas de impacto social en el país (Ministerio de Desarrollo Social, 2011).

El uso de prótesis entre los grupos presenta una diferencia significativa pues el programa GES en los 
pacientes casos permite un acceso privilegiado a la rehabilitación protésica, esto ha determinado una gran cantidad de recursos públicos y privados destinados para el GES, que en la práctica para el sector público, se traduce en una absoluta priorización al grupo GES en desmedro de cualquier otro tipo de paciente de otras edades o con otro tipo de necesidades no cubiertas por el GES (Superintendencia de Salud, 2011b).

En la evaluación de la satisfacción basada en la calidad de servicio percibido, el resultado muestra, por un lado, que la política de salud de cumplir con el objetivo sanitario planteado por el Ministerio de Salud de Chile de "Proveer de servicios de salud acordes a las expectativas de la población" (MINSAL, 2002) se cumple, pues los casos se muestran más satisfechos, ya que se le resuelven aspectos básicos de su salud oral como resolución de caries, efectuar extracciones, remplazo de dientes perdidos, resolviendo de esta manera necesidades de función, fonación y masticación, mejorando su autoestima.

El programa GES en Chile significa un avance, pues si se compara con otros países no se encuentra programas semejantes funcionando según nuestra búsqueda, en especial en el concierto latinoamericano. La división suiza de la OMS sobre la acción de la salud pública en la salud oral de adultos mayores en el mundo concluye que "la falta de apoyo financiero de los gobiernos y/o la falta de sistemas de pago de terceros hace de la seguridad social en salud oral poco económica". Luego agrega que los informes de los programas de promoción de salud que apuntan a los adultos mayores son raros y esto refleja la falta de política de salud oral", haciendo en consecuencia el caso del GES en Chile un programa de excepción.
Este programa de salud bucodental contribuye al objetivo sanitario del Ministerio de Salud: "enfrentar los desafíos derivados del envejecimiento de la población" (MINSAL, 2002) que busca responder a los "problemas de salud que no matan pero que disminuyen la calidad de vida, generando discapacidad, dolor y angustia", entre estos las necesidades bucodentarias más aún en un país con una creciente población senescente, que tiene un peso relativo cada vez mayor en Chile (INE) y que para el año 2025 representarán el 20\% de la población del país.

Entre las limitaciones de este estudio está el tamaño muestral del grupo control pues fue difícil el acceso a estos pacientes, a pesar de ello semantuvo la aleatorización. Además, los controles podrían haber perdido representatividad porque fueron pacientes que asistieron en forma espontánea a demandar atención odontológica y por tanto, su salud bucodental pudiera estar más protegida. Sin embargo, a pesar de ello, igual resultaron diferencias significativas en variables de salud oral a favor de la labor del programa GES.

Este estudio concluye que la reforma en salud en Chile a través del GES de Salud Oral Integral de 60 años, demuestra variar los parámetrosclínicos de los pacientes beneficiarios reflejado en un aumento significativo de prótesis y una disminución de los dientes cariados. Por otro lado, no logra una restitución ni una mantención de la salud periodontal, y el paciente no maneja los factores de riesgo, en consecuencia es esperable que en el tiempo las diferencias obtenidas al comparar los mismos grupos tiendan a desaparecer. Finalmente, la satisfacción del usuario es buena en los pacientes beneficiarios del GES en forma consistente, lo que convierte al programa GES en un activo de popularidad de la gestión de salud pública entre la población.

QUEZADA, P. C.; MUÑOZ, A. D.; CUETO, U. A. \& BARRAZA, S. A. Evaluation of explicit guarantees in oral health in 60-year-old Population. Int. J. Odontostomat., 7(2):319-326, 2013.

ABSTRACT: The Chilean reform created the Program of Explicit Guarantees in Health to safeguard the attention under guaranteed conditions of access, opportunity, financial protection and quality for prioritized situations such as oral health of the 60-year-old adult. Objective: To compare oral health and satisfaction between patient beneficiaries of the program of guaranteed oral health for 60 yearolds and non-beneficiaries. Methods: Case - control study. The participants were divided in cases $(n=48)$ and control group ( $n=28)$. Clinical examination gathering the variables: sex, age, systemic declared diseases, educational level, use of prosthesis, indexes COPD, CPITN and O'Leary. To measure satisfaction, SERVPERF (the multidimensional scale not weighted) was used. Inference analysis by means of test of significance (c2 Pearson, Fisher's, Mann-Whitney, Mood's Medians and Kolmogorov-Smirnov with a confidence level of $95 \%$ ) in the software SPSS19, Stata 10.1 and Minitab 15. In the oral measurements significant differences were found only in decay and COPD. There were no differences in the needs ofperiodontal treatment between the groups $(p$-value $=0.615)$. The presence of removable prosthesis was statistically greater in the group of cases (OR=4.4 [1.6-12.5]); $p$-value=0.006). The health reform known as GES Complete Oral Health for 60-year-old adults, demonstrates to change the clinical parameters of the patient beneficiaries, focused on restoration with a significant increase of prosthesis and a decrease of cavities, but it does not achieve maintenance of periodontal health and management of risk factors. User satisfaction was good among the beneficiaries of the program.

KEY WORDS: oral health program, elderly, diagnosis, oral, dental care for elderly, patient satisfaction. 


\section{REFERENCIAS BIBLIOGRÁFICAS}

Badenier, O.; Moya, R. \& Cueto, A. Estudio de prevalencia de Enfermedades Bucodentales y necesidades de tratamiento en la V región. Universidad de Valparaíso, Chile. Santiago, FONIS, 2007.

CELADE (Centro Latinoamericano y Caribeño de Demografía). División de Población de la CEPAL. El envejecimiento y las personas de edad. Indicadores sociodemográficos para América Latina y el Caribe. 2010. Disponible en: http://www.eclac.cl/publicaciones/xml/5/ 35915/L2987.pdf

CORMUVAL. Corporación Municipal de Valparaíso. Área Salud, Departamento de Estadística. Resumen altas integrales 60 años, Valparaíso, Corporación Municipal, 2009.

Cronin, J. \& Taylor, S. SERVPERF versus SERVQUAL: Reconciling Performance-Based and Perceptions-MinusExpectations Measurement of Service Quality. J. Market., 58(1):125-31, 1994.

Helft, M.; Cardash, H. S.; Rones, B. \& Ben-Ur, Z. The prevalence of denture related injuries in patients resident at two Israeli geriatric hospitals. Gerodontology, 5(2):1237, 1986.

Hernández Sampieri, R. Metodología de la Investigación. $4^{a}$ ed. México D.F., McGraw-Hill, 2006.

INE (Instituto nacional de estadísticas). Población adulta mayor en el bicentenario, Boletín Informativo del Instituto Nacional de Estadísticas, 2010. Disponible en: http:/ /www.ine.cl/filenews/files/2010/septiembre/pdf/ adulto_mayor_web_01-09-10(1).pdf

Mariño, R. J.; Cueto, A.; Badenier, O.; Acevedo, R. \& Moya, $R$. Oral health status and inequalities among ambulant older adults living in central Chile. Community Dent.Health, 28(2):143-8, 2011.

Mariño, R. La salud bucodental de los ancianos: realidad, mitos y posibilidades. Boletín de la Oficina Sanitaria Panamericana (OSP), 116(5):419-26, 1994.

MINSAL. Ministerio de Salud. Objetivos sanitarios para la década 2000-2010. El Vigía: Boletín de vigilancia en salud pública de Chile, 5(15):3-12, 2002.

MINSAL (Ministerio de Salud). Guía clínica Salud Oral Integral para Adultos de 60 años, Santiago, Ministerio de Salud, 2011. Disponible en: http://www.redsalud.gov.cl/ archivos/guiasges/saludoral60.pdf

Ministerio de Desarrollo Social. Chile Solidario. Noticias.
Santiago de Chile, Ministerio de Desarrollo Social, 2011. Disponible en: http://www.chilesolidario.gob.cl/not/ not_int1.php?id=415

Moreno, E. \& Miles, J. Instituto Nacional de Estadísticas. Adultos mayores por regiones, comunas y porcentajes. 2002. Disponible en: http://www.ine.cl/canales/ chile_estadistico/estadisticas_sociales_culturales/ adultosmayores/pdf/mayorescenso2002.pdf.

Organización Mundial de la Salud (OMS). Encuestas de Salud Bucodental. Métodos Básicos. $4^{\mathrm{a}}$ ed. Ginebra, OMS,1997.

Petersen, P. E.; Kandelman, D.; Arpin, S. \& Ogawa, H. Global oral health of older people-call for public health action. Community Dent. Health, 27(4 Suppl. 2):257-67, 2010.

Rojas, C.; Segovia, J.; Raccoursier, V.; Godoy, J. \& Lopetegui, M. S. Estado Periodontal y Necesidad de Tratamiento en Pacientes GES 60 Años de Villa Alemana. Rev. Clin. Periodoncia Implantol. Rehabil. Oral, 3(2):86-9, 2010.

Superintendencia de Salud. Casos GES acumulados a Marzo de 2011. Santiago, Gobierno de Chile, 2011a. Disponible en: http://www.supersalud.gob.cl/documentacion/ 569/w3-article-7127.html

Superintendencia de Salud. Informe final estudio 1879/08: Evaluación del acceso, equidad en el acceso e impacto de la implementación del Régimen de Garantías Explícitas de Salud. Santiago de Chile, Superintendencia de Salud, 2011b. Disponible en: http:// www.supersalud.gob.cl/documentacion/569/articles4332_recurso_1.pdf

Dirección para Correspondencia:

Daniela Muñoz Ávila

Subida Carvallo 211

Playa Ancha, Valparaíso

CHILE

Teléfono: $56-322508568$

Fax: $\quad$ 56-32 2508534

Email: daniela.munoz.avila@gmail.com

Recibido : 07-02-2013

Aceptado: 04-08-2013 